\title{
Liquid Crystalline Period Variations in Self- Assembled Block Copolypeptides-Surfactant Ionic Complexes
}

\author{
Chaoxu Li, Jingguo Li, Xiuqiang Zhang, Afang Zhang, Raffaele Mezzenga*
}

We investigate the complexation of ampholytic poly( $N$-isopropylacylamide)-block-poly(L-glutamic acid)-block-poly(L-lysine) (PNiPAM-b-PLG-b-PLLys) triblock copolymers and PNiPAM-block-(PLG-co-PLLys) diblock copolymers with counter charged anionic and cationic surfactants. Both triblock and diblock copolymers are able to selectively form complexes through either L-glutamic acid-cationic surfactant or L-lysine-anionic surfactant ionic pairs, depending on the protonated or deprotonated states of the ampholytic peptide units. The complexes show ordering at multiple length scales: i) the block copolymer length scale $\left(10^{1} \mathrm{~nm}\right)$, ii) the liquid crystalline length scale $\left(10^{\circ} \mathrm{nm}\right)$, and, iii) the peptidic secondary structures length scale $\left(10^{\circ} \mathrm{nm}\right)$. We show that the liquid crystalline period can be tuned by varying the random/block copolypeptide architectures and the composition of the ampholytic amino acid species.

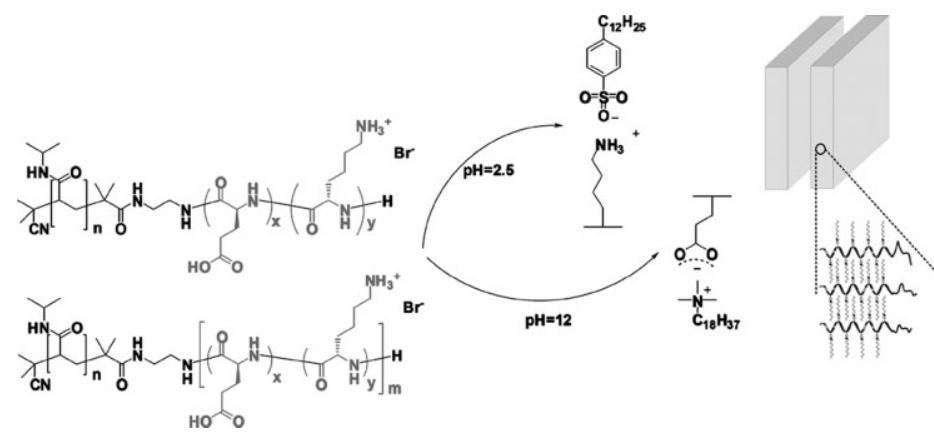

\section{Introduction}

Block copolypeptides have received a great deal of attention recently since they can be used as building blocks for designing functional nanostructured materials, ${ }^{[1]}$ for their

C. X. Li, R. Mezzenga

Department of Physics and Fribourg Center for Nanomaterials, University of Fribourg, Ch. du. Musée 3, CH-1700 Fribourg,

Switzerland

E-mail: raffaele.mezzenga@unifr.ch

J. Li, X. Zhang, A. Zhang

School of Materials Science and Engineering, Zhengzhou

University, China

R. Mezzenga

Néstlé Research Center, Vers-Chez-Les-Blanc, 1000 Lausanne 26, Switzerland

E-mail: raffaele.mezzenga@rdls.nestle.com appealing biocompatibility and their ability of folding into $\alpha$-helical or $\beta$-sheet secondary structures. ${ }^{[2-3]}$ The interplay of immiscibility between the blocks and organization of polypeptides leads to controllable hierarchical self-assembly at multiple length scales. ${ }^{[4-5]}$ The rod-like block arising from the $\alpha$-helical polypeptide secondary structures is particularly interesting due to its inter-rod liquid crystalline packing at the $10^{\circ} \mathrm{nm}$ length scale. Supramolecular incorporation of side-chains into one or several blocks of the block copolypeptides gives more flexibility for controlling the resulting hierarchical nanostructures and functionalities, ${ }^{[6]}$ since the final block copolymers show order at several length scales: the block copolymer structures (around $10^{1} \mathrm{~nm}$ ), the liquid crystalline structures of mesogen side-chains (around $10^{\circ} \mathrm{nm}$ ) and secondary structures of polypeptides (around $10^{\circ} \mathrm{nm}$ ). It is expected that self-assembly at different length scales can provide new routes to design functional materials. ${ }^{[7-11]}$ 
It is now well assessed that surfactants can be physically bonded to polypeptide backbones to achieve self-assembly in the solid state. ${ }^{[12-13]}$ Application of ionic interactions requires both the charged surfactants and oppositely charged polypeptides, most often poly(L-glutamic acid) (PLG) ${ }^{[14-15]}$ and poly(L-lysine) (PLLys). ${ }^{[16]}$ By combining in aqueous solutions the polypeptide and counter charged surfactant, a complex comb-like polymer precipitates, leading to a layered liquid crystalline structure in the solid state, similar to other conventional polyelectrolytesurfactant complexes. The period of the layers is expected to depend on both the structure of the surfactants as well as the secondary structures of the polypeptides. However, incorporation of the above polypeptide-surfactant complexes into block copolymers has seldom been explored so far. In "rod-comb" block copolymer poly( $\gamma$-benzyl-Lglutamate)-block-PLLys (PBLG-b-PLLys), where protonated PLLys were complexed with anionic surfactants, either hexagonal or lamellar arrangement of surfactant tails was reported within the PLLys-surfactant block at $10^{\circ} \mathrm{nm}$ length scale. $^{[17]}$ In poly(ethylene oxide)-block-poly-(L-glutamic acid) (PEO-b-PLG) where anionic PLG was complexed with cationic primary alkylamines, Hammond et al. found a well-ordered lamellar arrangement of alkyl tails in the PLGsurfactant block, leading to a hierarchical self assembly at the surfactant $\left(10^{0} \mathrm{~nm}\right)$ and block copolymer $\left(10^{1} \mathrm{~nm}\right)$ length scales. ${ }^{[18]}$ More recently, hierarchical ordering over six different length scales was found in triblock copolymer PLLys- $b$-PBLG- $b$-PLLys where cationic PLLys was complexed with anionic 2'-deoxyguanosine 5'-monophosphate. ${ }^{[19]}$

In this study, we report on the self-assembly of block and random copolypeptides-surfactant ionic complexes, in which the two different polypeptides have an ampholytic behavior. The hierarchical construction of the complexed systems leads to ordering at length scales of the block copolymer, the liquid crystalline and the peptidic secondary structures. The novelty of the study partially relies on the fact that in order to control the liquid crystalline period, rather than varying the surfactant tail length, as is found most often in the literature, we exploit the $\mathrm{pH}$-selective response of the poly(t-glutamic acid) and poly(t-lysine). Due to the ampholytic property of the compound, ${ }^{[20]}$ its charged states can be controlled from protonated to deprotonated by simply varying the $\mathrm{pH}$, leading to selective binding to anionic or cationic surfactants, respectively. We compare the resulting liquid crystalline period variation for random and block copolymer architectures and discuss the physical mechanisms responsible for these variations.

\section{Experimental Part}

\section{Synthesis of Block Copolymers}

Poly( $N$-isopropylacylamide)-block-poly[(L-glutamic acid)-co-(L-lysine)] [PNiPAM- $b$-(PLG-co-PLLys)] was prepared according to a previous report. $^{[20]}$ PNiPAM- $b$-PLG- $b$-PLLys was prepared by ring-opening polymerization of lysine anhydride from PNiPAM- $b$-PBLG according to another previous paper. ${ }^{[21]}$

\section{Complex Preparation}

Complexes were formed by mixing aqueous solutions of surfactant and polymer at a 1:1 stoichiometric ratio (surfactant/charged monomer). The block copolymer solution (ca. $0.5 \mathrm{wt}$. $\%$ ) was adjusted to a $\mathrm{pH}$ of approximately 12 in the case of complexation of polyglutamic acid with octadecyltrimethylammonium bromide (C18TAB) and approximately 2.5 in the case of complexation of polylysine with sodium dodecylbenzyl sulfonate (SDBS). The surfactant solution (0.5 wt.-\%) with the same $\mathrm{pH}$ was added slowly to the corresponding polymer solution under vigorous stirring. The precipitated complexes were collected by centrifugation, washed with water of the same $\mathrm{pH}$ as that used for the complexation, and dried thoroughly in a high vacuum annealing oven $\left(10^{-7} \mathrm{mBar}\right)$.

\section{Characterization}

Infrared spectra were collected on a Bruker Tensor 27 Fouriertransform infrared (FTIR) spectrometer in attenuated total reflection (ATR) mode, using a ZnSe crystal. The solid samples were pressed directly onto the crystal and spectra were recorded in the range $600-4000 \mathrm{~cm}^{-1}$ by averaging 32 spectra with $2 \mathrm{~cm}^{-1}$ resolution.

Small and wide-angle X-ray scattering (SWAXS) diffractograms were recorded using an Anton-Par SAXSess system. The system uses a sealed tube $\mathrm{Cu} \mathrm{Ka}(\lambda=0.154 \mathrm{~nm})$ source with a sample-todetector (image plate) distance of $26 \mathrm{~cm}$, which gives an effective $q$-range of $0.1-25 \mathrm{~nm}^{-1}$. Here $q$ is the scattering vector defined as $q=4 \pi \sin (\theta) / \lambda$, with $2 \theta$ being the scattering angle.

Transmission electron microscopy (TEM) images were obtained with a Philips TEM (CM 100) instrument operating at a voltage of $80 \mathrm{kV}$. After being glued on microtome sample holder tips, samples were cryo-sectioned by a diamond knife with a Leica Ultracut UCT ultramicrotome at ca. $-20^{\circ} \mathrm{C}$. Sections with a thickness of $50 \mathrm{~nm}$ were collected onto 600 mesh copper grids and then stained by $\mathrm{RuO}_{4}$ for $20 \mathrm{~min}$.

\section{Results and Discussion}

All the block copolypeptides investigated bear a 360 unit PNiPAM block in common (Figure 1). PNiPAM-b-(PLG-coPLLys) contains a random copolypeptide block from L-glutamic acid and L-lysine, while PNiPAM- $b$-PLG- $b$-PLLys possesses a poly(L-glutamic acid) block and a poly(L-lysine) block. Since the glutamic acid unit has an average $\mathrm{p} K_{\mathrm{a}}$ of 4.05 and the lysine unit 10.54, the cationic and anionic charged states of the block copolymer solutions can be easily adjusted by tuning the $\mathrm{pH}$. At $\mathrm{pH}<4.05$, due to protonation, the glutamic acid units are mostly neutralized and the lysine units are positively charged; this state allows selectively the protonated lysine units to be complexed by 

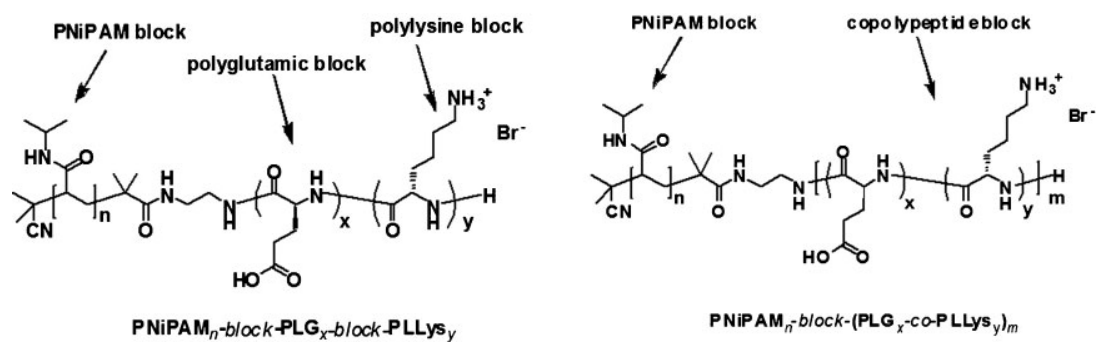

PNiPAM $_{n}$-block- $\left(\text { PLG }_{x}-\text { co-P LLys }_{y}\right)_{m}$

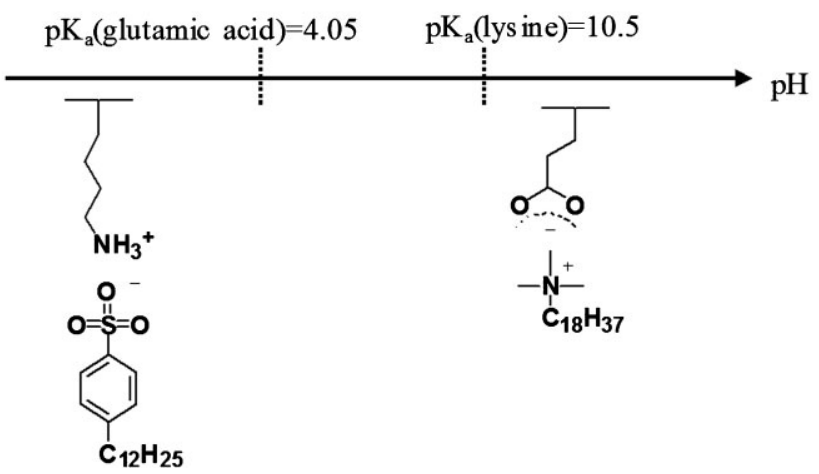

Figure 1. Chemical structures of block copolypeptides used in this study and pathway for synthesis of complexes.

negatively charged dodecylbenzyl sulfonate groups. At $\mathrm{pH}>10.05$, due to deprotonation the lysine units are neutralized and the glutamic acid units are negatively charged; this state allows the deprotonated glutamic acid units to be selectively complexed with positively charged octadecyltrimethylammonium groups. It is well established in the literature for polyelectrolyte-surfactant ionic complexes, ${ }^{[22-23]}$ that the ionic complexation at a 1:1 ratio of surfactant/charged monomer typically results in a

a)

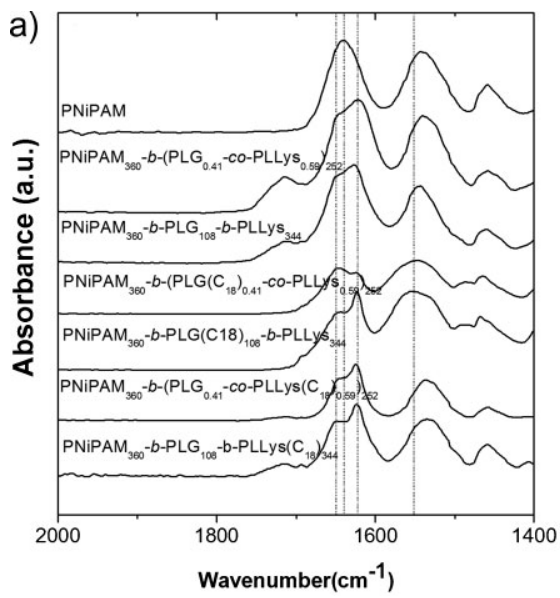

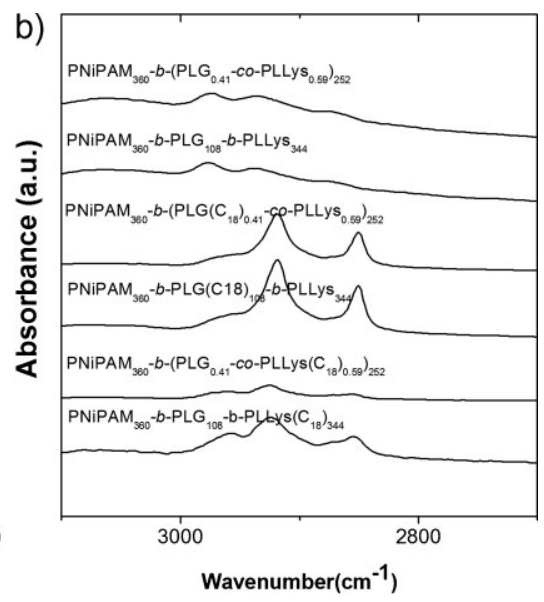

Figure 2. FTIR spectra of PNiPAM $360-b-\left(\mathrm{PLG}_{0.41}-\text { CO- } \mathrm{PLLys}_{\mathrm{O} .59}\right)_{252}$ and $\mathrm{PNiPAM}_{360}-b-\mathrm{PLG}_{108}$ $b$-PLLys ${ }_{344}$ before and after complexation with SDBS and C18TAB, in the 1400 to 2 $000 \mathrm{~cm}^{-1}$ (a) and $2700-3100 \mathrm{~cm}^{-1}$ (b) regions. The PNiPAM spectrum is given as a reference. spectrum of PNiPAM is reference. Although PNiPAM itself shows two main absorption peaks $\left(1640\right.$ and $1543 \mathrm{~cm}^{-1}$ ) between 1500 and $1800 \mathrm{~cm}^{-1}$, PNiPAM $_{360}-b-\left(\text { PLG }_{0.41}-\text { CO}^{-P_{L L y s}}{ }_{0.59}\right)_{252}$ and PNiPAM $_{360}-b-$ PLG $_{108}-b$-PLLys 344 and their complexes are all characterized by vibrations at 1623 and $1650 \mathrm{~cm}^{-1}$, which indicate that these peaks result from the secondary structures of polypeptide blocks, rather than from PNiPAM. In other words, $\alpha$-helix and $\beta$-sheet secondary structures were found to coexist in all the six samples. In this respect, the existence of the $\alpha$-helix was further confirmed by the peak (or shoulder in some cases) at $1550 \mathrm{~cm}^{-1}$. Rather unexpectedly considering the high molecular weight of the peptide blocks and with the exception of PNiPAM $_{360}-b$-[PLG(C18) $0.41^{-C O}$-PLLys 0.59$]_{252}$, which shows a stronger vibration at $1650 \mathrm{~cm}^{-1}$ than at $1623 \mathrm{~cm}^{-1}$, all other samples present much stronger vibration at $1623 \mathrm{~cm}^{-1}$ than at $1650 \mathrm{~cm}^{-1}$, indicating that $\beta$-sheets are the dominant secondary structure, and moreover, this domination is not influenced by either the topology of the block copolymers (triblock vs. diblock) or the complexation process. The fact that these trends occur not only on PNiPAM- $b$-(PLGco-PLLys) but also on the PNiPAM- $b$-PLG- 
$b$-PLLys, suggests that the unexpected balance in secondary structures does not originate from the neighboring of PLG and PLLys amino acids along the random copolymer block. Only in the case of

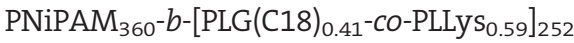
does the pronounced $\alpha$-helix fraction suggest that complexation can stabilize the $\alpha$-helix with respect to the $\beta$-sheet secondary structure. Figure $2 b$ shows the IR absorptions within the range 2800 to $3000 \mathrm{~cm}^{-1}$. The peaks at 2918 and $2850 \mathrm{~cm}^{-1}$ suggest that the alkyl tails in the surfactants are mainly in the extended conformation.

The presence of polyelectrolytesurfactant complexes in both random and block copolypeptides with coexisting secondary structures allows a very interesting study to be performed on the variation of liquid crystalline period with the molar fraction of bound surfactants. This effect has already been studied for hydrogen-bonded polymer-surfactant complexes in which non-stochiometric complexation can easily be achieved starting from non-stoichiometric monomer/surfactant feeding ratios. ${ }^{[25-}$ ${ }^{26]}$ In ionic complexes, however, this can only be studied using ampholytic copolymers or random copolymers in which one monomer is inert to complexation. ${ }^{\left[{ }^{[7]}\right.}$ Indeed, as mentioned above, in counter-charged homopolymersurfactant systems the final stoichiometric ratio is typically maintained unaltered in the final complexes independently of the feeding ratio. ${ }^{[22]}$ Furthermore, no study on coampholytic random copolymers with ordered secondary structures has been performed to date, to the best of our knowledge.

The hierarchical structures of the complexes were characterized by both WAXS and TEM. As shown in Figure 3, neither of the block copolymers showed peaks before complexation, which demonstrates that no ordered periodic arrangement was present within the pristine polypeptide chains. This is not unexpected due to the complicated secondary structures of polypeptide segments and the mutual strong inter-molecular hydrogen bonding between the $-\mathrm{COOH}$ and $-\mathrm{NH}_{2}$ groups of PLG and PLLys, respectively. However, complexation of any of the two amino acids virtually suppressed intramolecular hydrogen bonding, leaving peptidic hydrogen bonds as the only driving force to the formation of secondary structures. This is reflected both on the long-range microphase separation of the block copolymers (see TEM inset in Figure 3) and on the liquid crystalline structures appearing on the medium angle $x$-ray scattering profiles. Indeed, after complexation all the samples show two peaks spaced as 1:2 characteristic of the lamellae structure (Figure 3). The same structure has previously been reported for other stoichiometric polypeptide-surfactant complexes. ${ }^{[13,28]}$ Particularly, PNiPAM ${ }_{360^{-}}$ $b$-PLG(C18) ${ }_{108}$ - $b$-PLLys ${ }_{344}$ gives a lamellae spacing of $3.81 \mathrm{~nm}$, which is very close to the value of $3.93 \mathrm{~nm}$ reported in the literature for the nearly identical PLG-C18 complexes. ${ }^{[14]}$ Also, its very pronounced second-order peak indicates sharply defined lamellar interfaces, consistent with the appearance of crystallization of the surfactant tails which can be inferred by the crystallization peak at $15.10 \mathrm{~nm}^{-1}$. Notably, if the alkyl tail were fully extended and in a perfectly interdigitated configuration, giving a length of C18TAB of $23.8 \AA$, the PLG layers would result in a width of $14.3 \AA$, which is close to the value $13.6 \AA$ reported for the diameter of the rod-like PLG chain in an $\alpha$-helical conformation. ${ }^{[15]}$ Therefore, this lamellar structure most probably consists of alternated monolayers of polypeptides and monolayers of interdigitated surfactants. Thus, it can also be easily argued that the $\alpha$-helical secondary structure in this block is essentially associated with the PLG block, and the $\beta$-sheet structure with the PLLys block. Comparably, PNiPAM $_{360^{-}} b$-[PLG(C18) $0.41^{-}$-CO-PLLys 0.59$]_{252}$ has a lamellar structure with a period of $4.49 \mathrm{~nm}$, which is much larger than the $3.81 \mathrm{~nm}$ period observed for $\mathrm{PNiPAM}_{360^{\circ}} b$ PLG(C18) ${ }_{108}-b$-PLLys ${ }_{344}$. Furthermore, its less pronounced second-order peak and the absence of crystallization peak of surfactant tails suggest relatively more poorly organized surfactant layers. Although the increase of periodicity at sub-stoichiometric polymer/surfactant ratios is a wellknown trend observed in hydrogen-bonded systems, ${ }^{[25-26]}$ it is surprising to see this effect also in polypeptidic systems with ordered secondary structures. In hydrogen bonded systems the period is predicted to increase linearly with $(1-x)$ where $x$ is the molar fraction (with respect to stoichiometry) of bound surfactants; this has been argued to be the result of the increased folded cross-linked homopolymer backbone, which can thus reduce extra 
interfacial energy arising from uncomplexed binding sites. In the present case, however, the polymer backbone effective thickness is expected to have a reduced flexibility and be somehow "frozen" by the secondary structure, as also consistent with FTIR spectroscopy data shown in Figure 2.

We infer that the increased lamellar periodicity result from the random occurrence of lysine units in the polypeptide block. This is expected to somehow have a "softening effect" of the secondary structure, so that some increased degree of folding and cross section thickening may occur in the polypeptide backbone. Also, a less pronounced degree of interdigitation, which is consistent with medium and wide angle scattering profiles for the complexed random copolypeptide, may contribute to the observed increase in periodicity. This scheme, may also explain the differences in periodicity for the lamellar structures of $\mathrm{PNiPAM}_{360}$ - $b$-[PLG $\mathrm{PL}_{0.41}$-CO-PLLys(C18) 0.59$]_{252}$

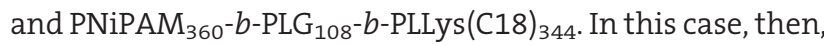
the much reduced difference in periodicity $(3.83 \mathrm{~nm}$ vs. $3.67 \mathrm{~nm}$ for PNiPAM 360 - $b$-[PLG P.41 -CO-PLLys(C18) 0.59$]_{252}$ and PNiPAM $_{360}-b-$ PLG $_{108}-b$-PLLys(C18) 344 , respectively), can simply arise from the higher complexation molar ratio surfactant/amino acid (0.59 compared to 0.41 in PNiPAM $_{360}-b$-[PLG 0.41 (C18)-co-PLLys 0.59$]_{252}$ ).

In summary, we have shown that ampholytic PNiPAMblock-PLG-block-PLLys triblock copolymers and PNiPAMblock-(PLG-co-PLLys) diblock copolymers constitute a remarkable system for ionic complexation with countercharged low-molecular-weight surfactants to generate hierarchical structures, where microphase separation, liquid crystalline interactions and peptidic secondary structures play a synergistic effect to define the final selfassembled structures. While complexation allows preserving the overall secondary structure of the peptide in both block and random copolymers, the control of the liquid crystalline period can be adjusted by the pristine composition of the ampholytic amino acid species.

Keywords: comb-like polymers; complexes; ionic complexes; polypeptides; side chain surfactants; supramolecular chemistry
[1] M. Lazzari, G. Liu, S. Lecommandoux, Block Copolymers in Nanoscience, Wiley-VCH, 2006, Chapter 6

[2] M. R. Caplan, D. A. Lauffenburger, Ind. Eng. Chem. Res. 2002, 41, 403.

[3] A. G. Walton, Polypeptides and Protein Structure, Elsevier, New York 1981.

[4] S. Lecommandoux, M. Achard, J. F. Langenwalter, H.-A. Klok, Macromolecules 2001, 34, 9100.

[5] J. Babin, D. Taton, M. Brinkmann, S. Lecommandoux, Macromolecules 2008, 41, 1384.

[6] M. R. Hammond, R. Mezzenga, Soft Matter 2008, 4, 952.

[7] O. Ikkala, G. ten Brinke, Science 2002, 295, 2407.

[8] R. Mezzenga, J. Ruokolainen, G. H. Fredrickson, E. J. Kramer, D. Moses, A. J. Heeger, O. Ikkala, Science 2003, 21, 1872.

[9] C. X. Li, A. D. Schluter, A. Zhang, R. Mezzenga, Adv. Mater. 2008, 20, 4530.

[10] O. Ikkala, G. ten Brinke, Chem. Commun. 2004, 2131.

[11] G. M. Whitesides, B. Grzybowski, Science 2002, 295, 2418.

[12] W. J. MacKnight, E. A. Ponomarenko, D. A. Tirrell, Acc. Chem. Res. 1998, 31, 781.

[13] S. Hanski, S. Junnila, L. Almasy, J. Ruokolainen, O. Ikkala, Macromolecules 2008, 41, 866.

[14] E. A. Ponomarenko, A. J. Waddon, D. A. Tirrell, W. J. Macknight, Langmuir 1996, 12, 2169.

[15] E. A. Ponomarenko, A. J. Waddon, K. N. Bakeev, D. A. Tirrell, W. J. Macknight, Macromolecules 1996, 29, 4340.

[16] E. A. Ponomarenko, D. A. Tirrell, W. J. Macknight, Macromolecules 1996, 29, 8751.

[17] S. Hanski, N. Houbenov, J. Ruokolainen, D. Chondronicola, H. Iatrou, N. Hadjichristidis, O. Ikkala, Biomacromolecules 2006, 7, 3379.

[18] M. R. Hammond, H. A. Klok, R. Mezzenga, Macromol. Rapid Commun. 2008, 29, 299.

[19] N. Houbenov, A. Nykänen, H. Iatrou, N. Hadjichristidis, J. Ruokolainen, C. F. J. Faul, O. Ikkala, Adv. Funct. Mater. 2008, 18, 2041.

[20] J. Li, T. Wang, D. Wu, X. Zhang, J. Yan, S. Du, Y. Guo, J. Wang, A. Zhang, Biomacromolecules 2008, 9, 2670.

[21] X. Zhang, J. Li, W. Li, A. Zhang, Biomacromolecules 2007, 8, 3557.

[22] N. Canilho, M. Sholl, H. A. Klok, R. Mezzenga, Macromolecules 2007, 40, 8374.

[23] N. Canilho, E. Kasëmi, A. D. Schlüter, J. Ruokolainen, R. Mezzenga, Macromolecules 2007, 40, 7609.

[24] E. R. Blout, A. Asadourian, J. Am. Chem. Soc. 1956, 78, 955.

[25] M. C. Luyten, G. O. R. Alberda van Ekenstein, J. Wildeman, G. ten Brinke, J. Ruokolainen, O. Ikkala, M. Torkkeli, R. Serimaa, Macromolecules 1998, 31, 9160

[26] J. de Wit, G. A. van Ekenstein, E. Polushkin, J. Korhonen, J. Ruokolainen, G. ten Brinke, Macromolecules 2009, 42, 2009.

[27] C. K. Nisha, P. Basak, S. V. Manorama, Langmuir, 2003, 19, 2947.

[28] E. A. Ponomarenko, D. A. Tirrell, W. J. MacKnight, Macromolecules 1998, 31, 1584 